CFD Letters

\title{
Exact Solution of Fractional Convective Casson Fluid Through an Accelerated Plate
}

\author{
Muhammad Nazirul Shahrim¹, Ahmad Qushairi Mohamad ${ }^{1,}{ }^{*}$, Lim Yeou Jiann ${ }^{1}$, Muhamad Najib \\ Zakaria $^{1}$, Sharidan Shafie ${ }^{1}$, Zulkhibri Ismail ${ }^{2}$, Abdul Rahman Mohd Kasim² \\ Department of Mathematical Sciences, Faculty of Science, Universiti Teknologi Malaysia, 81310 Johor Bahru, Johor, Malaysia \\ 2 Centre for Mathematical Sciences, Universiti Malaysia Pahang, Lebuhraya Tun Razak, 26300 Gambang, Kuantan Pahang, Malaysia
}

\section{ARTICLE INFO ABSTRACT}

\section{Article history:}

Received 29 February 2021

Received in revised form 25 June 2021

Accepted 29 June 2021

Available online 30 June 2021

\section{Keywords:}

Casson fluid; Caputo derivative; Laplace transform; Wright function; Heat transfer

\begin{abstract}
Fractional derivative has perfectly adopted to model few physical phenomena such as viscoelasticity of coiling polymers, traffic construction, fluid dynamics and electrical networks. However, the application of the fractional derivatives for describing the physical characteristics of non-Newtonian fluid over a moving plate is still rare. In the present study, the effect of the Caputo fractional derivative on the Casson fluid flow which is induced by an accelerated plate is analytically analysed. The governing equations are initially transformed into dimensionless expressions by using suitable dimensionless variables. Then the Laplace transform method is utilized to calculate the exact solutions for the fractional governing partial differential equations. The obtained solutions are validated by comparing the results for specific case with the existing solutions in the literature. The impact of fractional parameter, Prandtl number, and time on the velocity and temperature profiles are graphically showed and discussed. The results depict that the temperature and velocity increase with the increment of fractional parameter and time. Interestingly, the velocity decreases at region near the plate but is enhanced at the area far away from the plate when the Casson fluid parameter is increased. This study is essential in understanding the factional nonNewtonian fluid flows which is more realistic in nature.
\end{abstract}

\section{Introduction}

Fractional derivatives have recently gained the attention of plenty of researchers due to their generality in covering derivatives and integrals of non-integer order. Various physical phenomena or natural situations such as viscoelasticity of coiling polymers, bioengineering, signal and image processing, rheology, fluid dynamics, traffic modeling, electric circuits, electrical networks, and stochastic processes have adapted the fractional calculus to study the influence of the memory effect and some essential parameters to the system. Imran et al., [1] studied the convection flow of Newtonian fluid by using two different fractional derivatives which are called Caputo and CaputoFabrizio. The solutions of the temperature, velocity, and concentration profiles were determined by

\footnotetext{
* Corresponding author.

E-mail address: ahmadqushairi@utm.my (Ahmad Qushairi Mohamad)
} 
using the Laplace transform method. The obtained results were illustrated graphically to compare and analyze the distinction between the two fractional derivatives.

Further, Imran et al., [2] demonstrated the application of Caputo time-fractional derivatives on Newtonian fluid flow with arbitrary velocity and Newtonian heating. The governed equations were firstly transformed into dimensionless form by using the given dimensionless variables. Then, the dimensionless equations were solved by using the Laplace transform technique. The authors found that the fractional parameter shows a significant influence on the velocity and temperature profiles. Imran et al., [3] extended the work done by Sheikh et al., [4] to investigate the convection flow of a Newtonian fluid in the existence of heat sink and chemical reaction. Similarly, the Laplace transform method was utilized to solve the governing dimensionless equations, and the results of the velocity, temperature, and concentration were depicted graphically. In the study, the authors have compared the results obtained by using Antagana-Baleanu and Caputo-Fabrizio approaches with the solutions determined by the classical model of the problem.

Besides that, Khan et al., [5] acknowledged the Caputo time-fractional derivative of a generalized Casson fluid with oscillating boundary conditions. The authors have calculated the closed-form expressions for the velocity and shear stress by using the Laplace transform technique. The influence of the fractional parameter, Casson parameter, and the oscillating frequency on the fluid velocity and shear stress were analyzed and discussed. Ali et al., [6] used the Caputo fractional derivative to study the blood flow, which is represented by a Casson fluid model, in a horizontal cylinder. The fluid flow was driven by the oscillating pressure gradient and the fluid was included with magnetic particles. The effects of the magnetohydrodynamics on the Casson fluid were analyzed and discussed by solving the fractional governing equations with the Laplace and finite Hankel transforms to obtain the exact solutions. The authors observed that the fluid model with fractional order behaves remarkably differently as compared to the ordinary model. Some recent interesting analytical study on the fluid problems can be found in previous studies [7-9].

Sheikh et al., [4] conducted a comparative study of the two approaches of fractional derivative which are Caputo-Fabrizio and Antagana-Baleanu fractional derivatives for the convection flow of Casson fluid over an infinite vertical flat plate coupled with heat and mass transport. Exact solutions for both cases were determined by using the Laplace transform methods and the solutions were compared graphically as well as in tabular form. The authors found that the velocities obtained via Antagana-Baleanu and Caputo-Fabrizio derivatives are identical for unit time. However, variation happens when time less than 1, and the differences are enhanced as time greater than 1 . Further, Sheikh et al., [10] extended the work by considering the effect of heat generation and first-order chemical reaction.

Casson fluid is a shear-thinning liquid and exhibits infinite viscosity at zero shear stress. The fluid behaves like a solid when there is yield stress applied to the fluid which is greater than the shear stress. However, the fluid starts to flow when the shear stress is greater than the applied yield stress. Examples of the Casson fluid include honey, soup, chocolate tomato sauce, jelly, slurries, molten polymers, toothpaste, paint, and blood. This kind of fluid model is found to have significant applications in food manufacturing, biomechanics, textiles, cosmetics, polymer processing industries, and pharmaceuticals. Saqib et al., [11] explored on heat and mass transfer process in the Casson fluid flow past an oscillating plate with chemical reaction and slip effect. The governing equations were converted into dimensionless form and then solved by using the Laplace transform method. The velocity, temperature, and concentration profiles were obtained and illustrated graphically.

Mahantesh et al., [12] investigated the convective flow of Casson fluid past an oscillating plate by taking non-coaxial rotation with quadratic density fluctuation as its boundary conditions. Firstly, the governing equations were transformed into a dimensionless form by utilizing some dimensionless 
variables. Then, the dimensionless momentum, heat, and mass equations were solved analytically by applying the Laplace transform technique. Nadeem et al., [13] investigated the boundary layer MHD flow of Casson fluid over an exponentially permeable shrinking sheet. The analytical solution of the problem was obtained by employing the Adomian decomposition method. The velocity distributions due to different interesting parameters were illustrated and analyzed. Nadeem et al., [14] studied the effect of magnetic field on the boundary layer three-dimensional Casson fluid flow over a stretching porous sheet. The authors found that the magnetic field, Casson fluid parameter, and porosity parameter reduce the velocity profiles in $\mathrm{x}$ - and $\mathrm{y}$ - directions.

Khan et al., [15] analyzed the effects of heat generation and chemical reaction on the MHD convection Casson fluid flow through a moving vertical plate in a porous medium. Besides, the influence of the Newtonian heating on the plate surface and thermal radiation were also considered in the study. Laplace transform method was utilized to determine the general. The effects of various parameters such as Casson fluid parameter, the permeability of the porous medium, chemical reaction parameter, heat generation parameter, buoyancy force parameter, magnetic parameter, and radiation parameter on the fluid flow were analyzed and graphically illustrated. Mohamad et al., [16] carried out a study on the flow of Casson fluid in a rotating channel disk. Similarly, the Laplace transform method was practiced by the authors. The effect of the rotating disk on the Casson fluid flow was analyzed and found that the primary velocity of the fluid decrease as the rotation parameter is increased while the secondary velocity is increased.

Alwawi et al., [17] acknowledged the MHD free convection flow of Sodium Alginate nanofluid on a solid sphere with prescribed wall temperature in the presence of a magnetic field. Sodium alginate is a fluid extracted from brown algae and it is a natural Casson fluid. The Keller-box method was utilized to compute the numerical solutions of the problem. The influence of nanoparticles volume fraction, Casson fluid parameter, magnetic parameter on velocity, temperature, local skin friction coefficient, and local Nusselt number was analyzed. Kumar et al., [18] studied the effect of the timedependent magnetohydrodynamic on the Casson fluid across an exponentially stretching curved sheet in the existence of an irregular heat sink/source. The governing equations were solved numerically and the influence of various dimensionless parameters on the fields of velocity and temperature were analyzed and discussed. The results reveal that the Casson parameter has enhanced the distribution of momentum, but an inverse development is observed for the curvature parameter.

Based on the literature reviews, many researchers have considered vertical and oscillating plates. To the best of the authors' knowledge, the free convection flow of fractional Casson fluid past an accelerated plate has not been considered yet so far. Therefore, the present study aims to determine the exact solutions of free convection flow of fractional Casson fluid through an accelerated plate by using the Laplace transform method. The effect of the various parameters such as Casson fluid parameter, fractional parameter, time, and Prandtl number on the fluid flow is analyzed and discussed.

\section{Problem Definition}

An unsteady free convection flow of an incompressible Casson fluid over an infinite accelerated vertical plate is considered. The flow being confined to $y>0$, where $y$ is the coordinate measured in the normal direction to the plate. Initially, for a time $t=0$, both the fluid and the plate are at rest with uniform temperature $T_{\infty}$. At the time $t>0$, the plate begins to accelerate in its plane according to a velocity $A t$, where the constant $A$ is the acceleration of the plate. At the same time, the plate temperature is raised to $T_{w}$, which is thereafter maintained constant. The velocity and temperature 
depend on space variable $y$ and time $t$. By taking unidirectional flow and the Boussinesq's approximation, the momentum and energy equations take the forms:

$$
\begin{aligned}
& \frac{\partial v^{*}(y, t)}{\partial t}=v\left(1+\frac{1}{\beta}\right) \frac{\partial^{2} v^{*}(y, t)}{\partial y^{2}}+g \beta_{T}\left(T-T_{\infty}\right) \\
& \rho C_{p} \frac{\partial T(y, t)}{\partial t}=K \frac{\partial^{2} T(y, t)}{\partial y^{2}}
\end{aligned}
$$

associated with initial and boundary conditions:

$$
\begin{array}{lll}
v^{*}(y, 0)=0, & T(y, 0)=T_{\infty}, & y>0, \\
v^{*}(0, t)=A t, & T(0, t)=T_{w}, & t>0, \\
v^{*}(\infty, t)=0, & T(\infty, t)=T_{\infty}, & t>0 .
\end{array}
$$

where $v^{*}=$ velocity component in $y$-direction, $\beta=$ Casson fluid parameter, $g=$ gravitational acceleration, $\beta_{T}=$ volumetric thermal coefficient of expansion, $\rho=$ fluid density, $C_{p}=$ specific heat capacity and $K=$ thermal conductivity parameter.

\subsection{Problem Solution}

The dimensional governing Eq. (1) and Eq. (2) are transformed into dimensionless equations. Next, by implementing the Laplace transform technique, the solutions are obtained. The governing equations are transformed into dimensionless equations by using suitable dimensionless variables:

$$
v=\frac{v^{*}}{(v A)^{\frac{1}{3}}}, \quad \xi=\frac{y A^{\frac{1}{3}}}{v^{\frac{2}{3}}}, \quad \tau=\frac{t A^{\frac{2}{3}}}{v^{\frac{1}{3}}}, \quad \theta=\frac{T-T_{\infty}}{T_{w}-T_{\infty}}
$$

The governing momentum and energy Eq. (1), Eq. (2) and boundary conditions Eq. (3) in the dimensionless form are

$$
\begin{array}{ll}
\frac{\partial v}{\partial \tau}=\left(1+\frac{1}{\beta}\right) \frac{\partial^{2} v}{\partial \xi^{2}}+G r \theta \\
\operatorname{Pr} \frac{\partial \theta}{\partial \tau}=\frac{\partial^{2} \theta}{\partial \xi^{2}} \\
v(\xi, 0)=0, & \theta(\xi, 0)=0, \\
v(0, \tau)=\tau, & \theta(0, \tau)=1, \\
v(\infty, \tau)=0, & \theta(\infty, \tau)=0 .
\end{array}
$$

Next, Eq. (4) and Eq. (5) are defined as Caputo fractional derivatives: 


$$
\begin{aligned}
& D_{t}^{\alpha} v(\xi, \tau)=\left(1+\frac{1}{\beta}\right) \frac{\partial^{2} v}{\partial \xi^{2}}+G r \theta \\
& \operatorname{PrD}_{t}^{\alpha} \theta(\xi, \tau)=\frac{\partial^{2} \theta}{\partial \xi^{2}}
\end{aligned}
$$

where $D$ is the differentiation operator and $\alpha$ is the fractional parameter, $\theta$ is the Casson fluid parameter, $G r$ is the Grashof number and Pr is the Prandtl number.

\subsection{Laplace Transform Technique}

Laplace transform is an integral transform method and is commonly used to solve the unsteady differential equations. By using the Laplace transform method, the partial differential Eq. (7) and Eq. (8) are transformed into a second order differential equations that are written as

$$
\begin{aligned}
& \left(1+\frac{1}{\beta}\right) \frac{\partial^{2} \bar{v}}{\partial y^{2}}(\xi, q)-q^{\alpha} \bar{v}(\xi, q)+G r \bar{\theta}=0, \\
& \frac{d^{2} \bar{\theta}}{d y^{2}}(\xi, q)-\operatorname{Pr}^{\alpha} \bar{\theta}(\xi, q)=0 .
\end{aligned}
$$

Eq. (9) and Eq. (10) are then solved by using the undetermined coefficient method and the solutions are presented as

$$
\begin{aligned}
& \bar{v}(\xi, q)=\frac{1}{q^{2}} e^{-\xi \sqrt{\gamma q^{\alpha}}}+\frac{1}{q} e^{-\xi \sqrt{\gamma q^{\alpha}}} \frac{\gamma G r}{q^{\alpha}(P r-\gamma)}-\frac{1}{q} e^{-\xi \sqrt{P r q^{\alpha}}} \frac{\gamma G r}{q^{\alpha}(P r-\gamma)} \\
& \bar{\theta}(\xi, q)=\frac{1}{q} e^{-\xi \sqrt{P r q^{\alpha}}} .
\end{aligned}
$$

Eq. (9) and Eq. (10) are then solved by implementing the inverse Laplace transform technique. The solutions of the velocity and temperature are written as:

$$
\begin{aligned}
& v(\xi, \tau)=\tau\left(2,-\frac{\alpha}{2},-\xi \sqrt{\frac{\beta}{\beta+1}} \tau^{-\frac{\alpha}{2}}\right)+\frac{\left(\frac{\beta}{\beta+1}\right)}{\operatorname{Pr}-\left(\frac{\beta}{\beta+1}\right)} G r \frac{\tau^{\alpha-1}}{r(\alpha)} \varphi\left(1,-\frac{\alpha}{2},-\xi \sqrt{\frac{\beta}{\beta+1}} \tau-\frac{\alpha}{2}\right)- \\
& \frac{\left(\frac{\beta}{\beta+1}\right)}{\operatorname{Pr}-\left(\frac{\beta}{\beta+1}\right)} \operatorname{Gr} \frac{\tau^{\alpha-1}}{r(\alpha)} \varphi\left(1,-\frac{\alpha}{2},-\xi \sqrt{\operatorname{Pr} \tau}-\frac{\alpha}{2}\right) \\
& \theta(\xi, \tau)=\varphi\left(1,-\frac{\alpha}{2},-\xi \sqrt{\operatorname{Pr}} \tau^{-\frac{\alpha}{2}}\right) .
\end{aligned}
$$

where $\varphi(*)$ is the Wright function. 


\section{Results and Discussion}

Eq. (11) and Eq. (12) are the closed form for the free convection flow of generalized fractional Casson fluid over an accelerated plate and the solutions are illustrated graphically by using MATHCAD software. The graphs are plotted with different values of embedded parameters to investigate the effect of the various parameters on the velocity and temperature profiles. For temperature profiles, the graphs are plotted with various embedded parameters to investigate the effect of embedded fractional parameter $\alpha$, Prandtl number Pr and time $t$ towards the temperature profiles which are shown in Figures 1-3. As for velocity profiles, the graphs are plotted with various fractional parameter $\alpha$, Casson fluid parameter $\beta$, Grashof number $\mathrm{Gr}$, and time $t$ and are shown in Figures 4-7. Meanwhile, validation of current solutions is displayed in Figure 8.

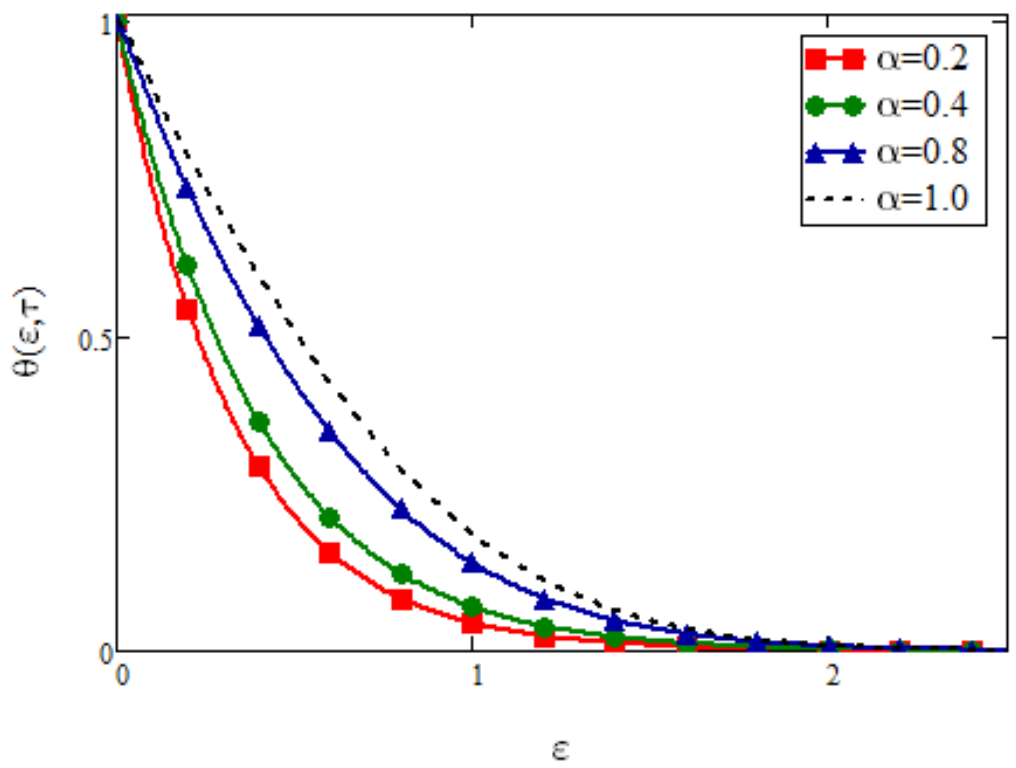

Fig. 1. Temperature profile with different $\alpha$

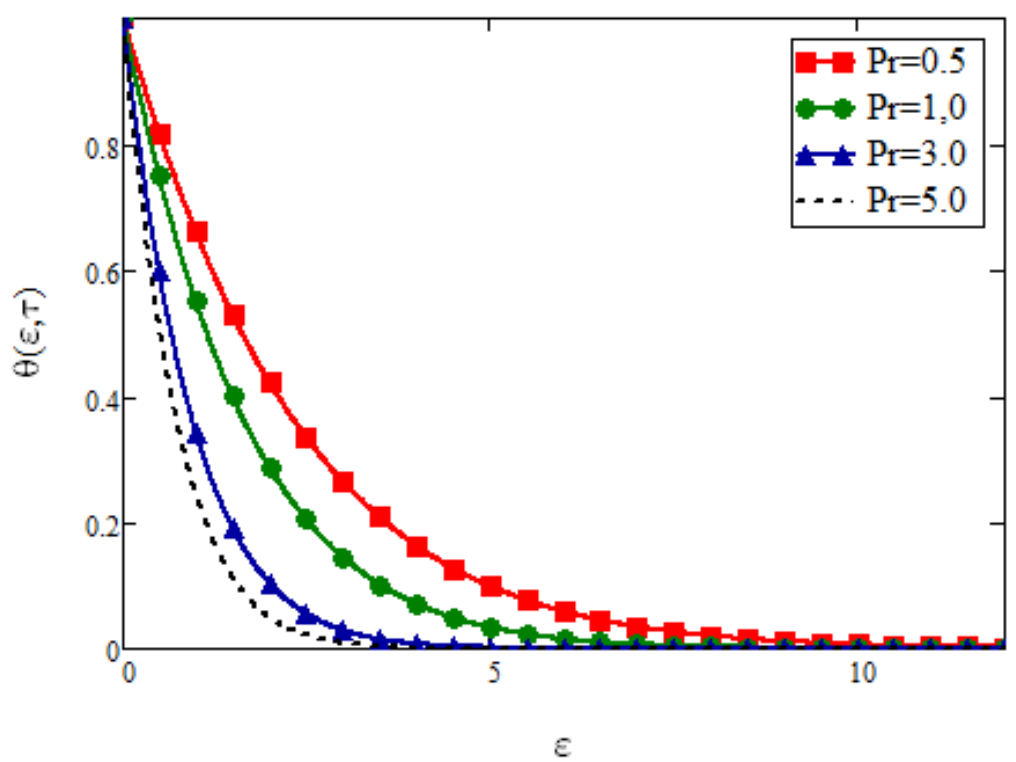

Fig. 2. Temperature profile with different $\mathrm{Pr}$ 


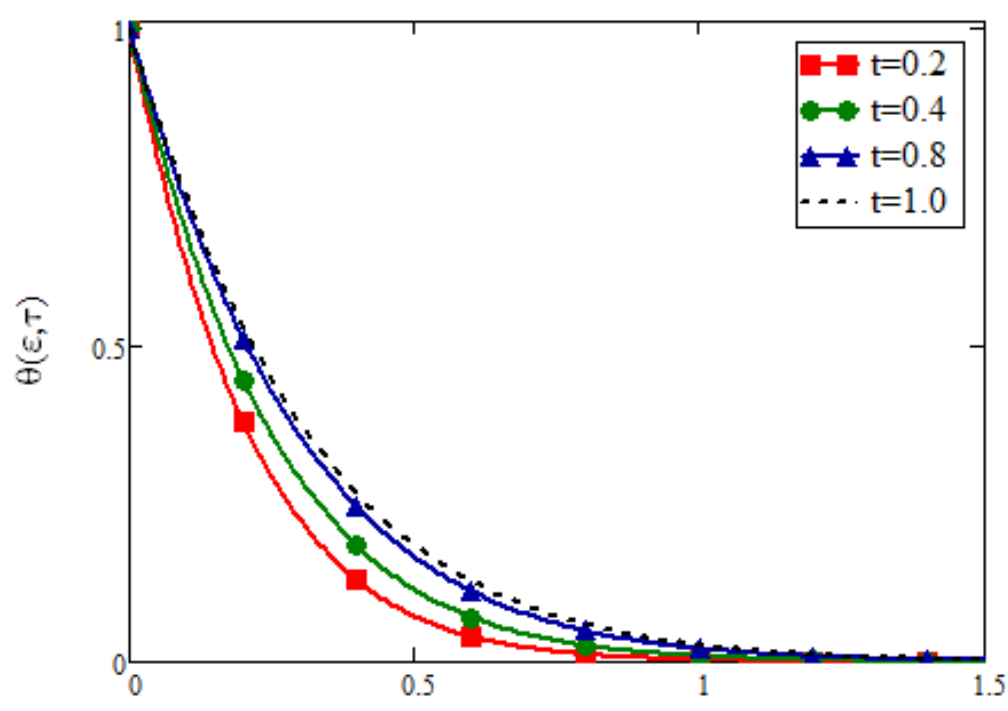

Fig. 3. Temperature profile with different $t$

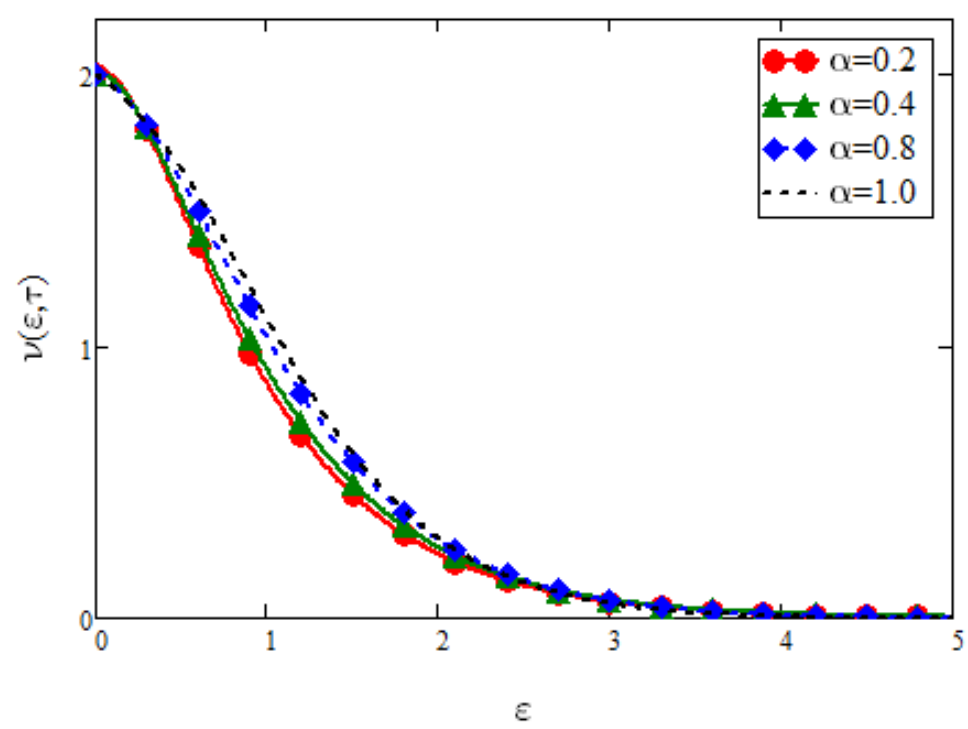

Fig. 4. Velocity profile with different $\alpha$

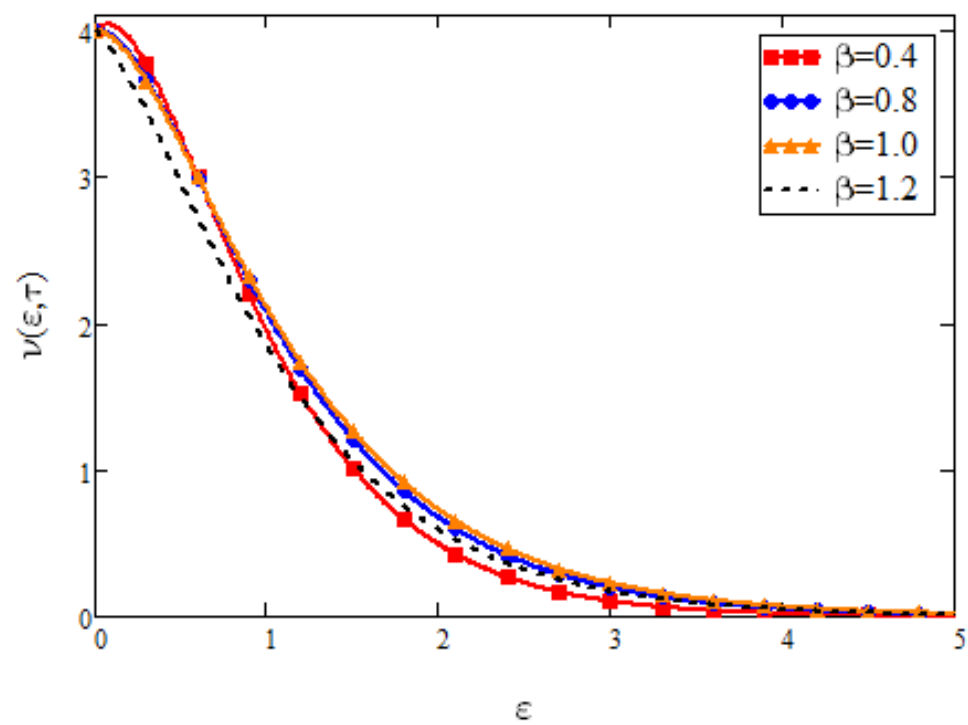

Fig. 5. Velocity profile with different $\beta$ 


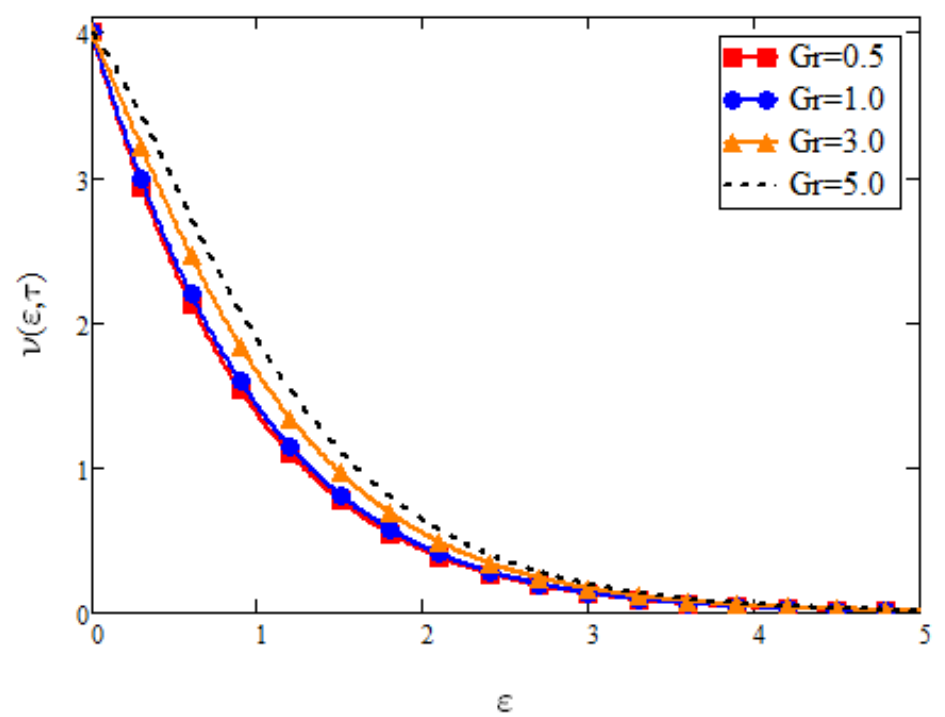

Fig. 6. Velocity profile with different $\mathrm{Gr}$

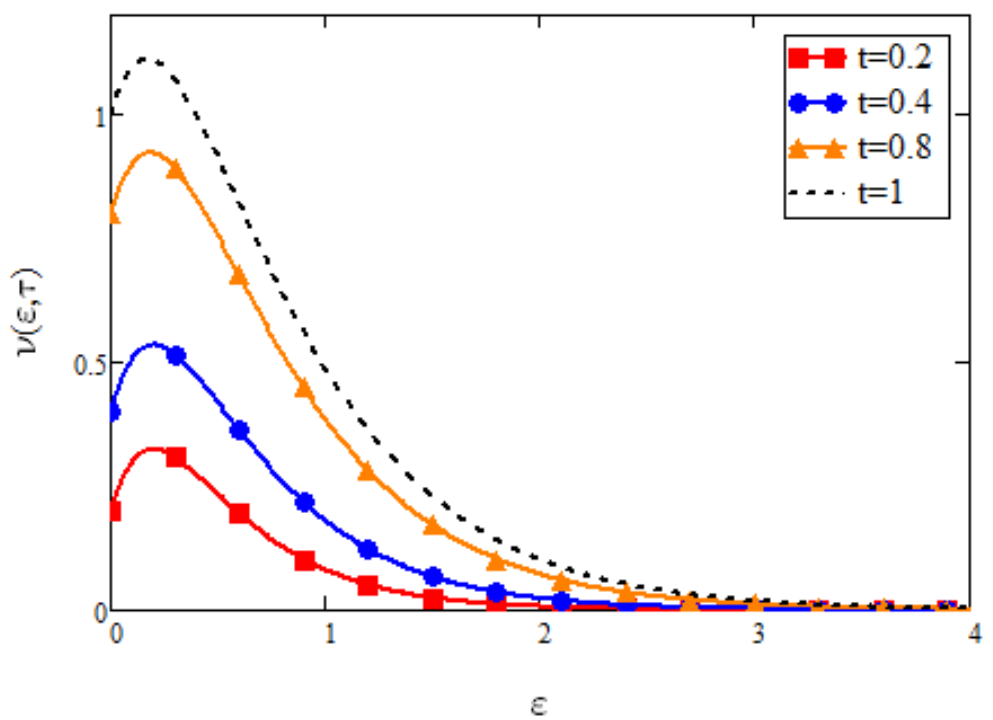

Fig. 7. Velocity profile with different $t$

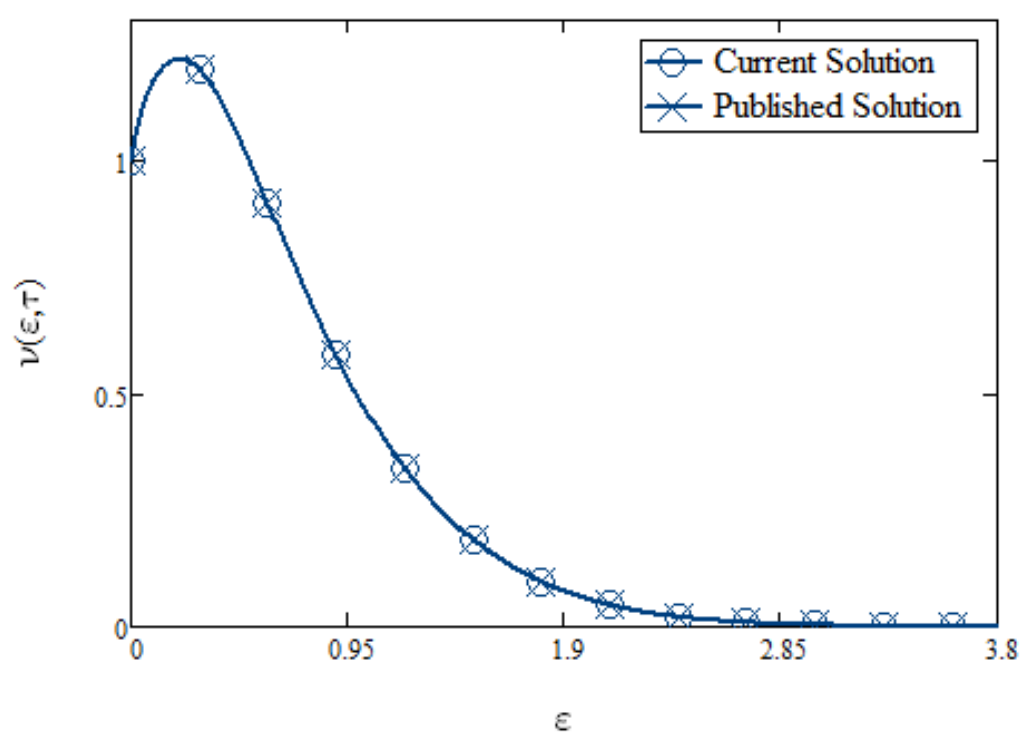

Fig. 8. Validated result with Ali et al., [19] 
Figure 1 and Figure 4 show the effect of fractional parameters on temperature and velocity profiles respectively. As shown in both figures, the temperature and velocity increase monotonically as increases. The results obtained here can be useful for some practical problems. By choosing an appropriate fractional mathematical model, the computed theoretical results can be used to estimate the expected outcome and the range for an experimental design. In Figure 2, the effect of the Prandtl number on the temperature distribution is presented. The graph depicts that the temperature profile decreases rapidly as the value of Pr increases. Prandtl number is defined as the ratio of momentum diffusivity to thermal diffusivity. Therefore, when Prandtl number Pr is small, the fluid moves slower than the heat transfer, in other words, the thickness of the thermal boundary layer is much bigger than the momentum boundary layer. So, heat can diffuse from the sheet faster for higher Pr fluids. However, for larger Prandtl number may reduce the thermal boundary layer thickness, and then reduce the thermal force to transport which consequently decreases the temperature profile.

Figure 3 and Figure 7 display the effect of time $t$ towards velocity and temperature profiles. As the value of $t$ increases, both velocity and temperature also rise up but at a different rate. In Figure 3, temperature increase gradually. Meanwhile, in Figure 7, the velocity increases dramatically. In general, the trend in both graphs shows that the temperature and velocity increase with increasing $t$. This trend can be explained as the value of $t$ increases, the energy produced from the fluid flow will eventually increase, this explained on the trend of the graphs. Furthermore, the effect of the Casson parameter on the velocity profile is shown in Figure 5 . The velocity experiences a decreasing trend at first and at some point, the velocity rises slowly. This is the characteristic of the Casson fluid. As the value increases, the shear stress of the fluid slowly overcomes the yield stress, and consequently the boundary layer thickness increase.

Figure 6 shows the effect of Grashof number Gr on the velocity profile. Gr signifies the relative effect of thermal buoyancy force on the viscous hydrodynamic force. From the observation, we can say that the value of velocity increases gradually as the value increases. This is because the Grashof number will enhance the buoyancy force of the flow and as a result, the velocity of the fluid flow will increase.

Finally, it is observed from Figure 8 that solutions from this study are in perfect agreement with published solutions from Ali et al., [19]. The limiting case of $t=1$ and $\omega=0$ were considered to validate findings.

\section{Conclusions}

The free convection flow of fractional Casson fluid past an accelerated plate has been investigated. The solutions of velocity and temperature were obtained by using the Laplace transform method. The effect of the various parameters such as Casson fluid parameter, fractional parameter, time, and Prandtl number on the fluid flow are discussed where the velocity increased when Gr and $t$ increased whereas velocity decreased when $\operatorname{Pr}$ and $\beta$ decreased. It is also concluded that solutions obtained from this study is valid.

\section{Acknowledgement}

The authors would like to acknowledge the Ministry of Higher Education Malaysia and Research Management Centre-UTM, Universiti Teknologi Malaysia (UTM) for financial support through vote numbers 17J98, FRGS/1/2018/STG06/UTM/02/4, FRGS/1/2019/STG06/UTM/02/22, and 08G33. 


\section{References}

[1] Imran, M. A., Shakila Sarwar, M. Abdullah, and I. Khan. "An analysis of the semi-analytic solutions of a viscous fluid with old and new definitions of fractional derivatives." Chinese journal of physics 56, no. 5 (2018): 1853-1871. https://doi.org/10.1016/j.cjph.2018.08.017.

[2] Imran, M. A., Nehad Ali Shah, Ilyas Khan, and Maryam Aleem. "Applications of non-integer Caputo time fractional derivatives to natural convection flow subject to arbitrary velocity and Newtonian heating." Neural Computing and Applications 30, no. 5 (2018): 1589-1599. https://doi.org/10.1007/s00521-016-2741-6.

[3] Imran, M. A., Maryam Aleem, M. B. Riaz, Rizwan Ali, and Ilyas Khan. "A comprehensive report on convective flow of fractional $(A B C)$ and $(C F)$ MHD viscous fluid subject to generalized boundary conditions." Chaos, Solitons \& Fractals 118 (2019): 274-289. https://doi.org/10.1016/j.chaos.2018.12.001.

[4] Sheikh, Nadeem Ahmad, Farhad Ali, Muhammad Saqib, Ilyas Khan, and Syed Aftab Alam Jan. "A comparative study of Atangana-Baleanu and Caputo-Fabrizio fractional derivatives to the convective flow of a generalized Casson fluid." The European Physical Journal Plus 132, no. 1 (2017): 1-14. https://doi.org/10.1140/epjp/i2017-11326-y.

[5] Khan, Ilyas, Nehad Ali Shah, and Dumitru Vieru. "Unsteady flow of generalized Casson fluid with fractional derivative due to an infinite plate." The European Physical Journal Plus 131, no. 6 (2016): 1-12. https://doi.org/https://doi.org/10.1140/epjp/i2016-16181-8.

[6] Ali, Farhad, Nadeem Ahmad Sheikh, llyas Khan, and Muhammad Saqib. "Magnetic field effect on blood flow of Casson fluid in axisymmetric cylindrical tube: A fractional model." Journal of Magnetism and Magnetic Materials 423 (2017): 327-336. https://doi.org/10.1016/j.jmmm.2016.09.125.

[7] Wahid, Nur Syahirah, Mohd Ezad Hafidz Hafidzuddin, Norihan Md Arifin, Mustafa Turkyilmazoglu, and Nor Aliza Abd Rahmin. "Magnetohydrodynamic (MHD) Slip Darcy Flow of Viscoelastic Fluid Over A Stretching Sheet and Heat Transfer with Thermal Radiation and Viscous Dissipation." CFD Letters 12, no. 1 (2020): 1-12.

[8] Ewis, Karem Mahmoud. "Analytical Solution of Modified Bingham Fluid Flow through Parallel Plates Channel Subjected to Forchheimer Medium and Hall Current Using Linearized Differential Transformation Method." Journal of Advanced Research in Numerical Heat Transfer 4, no. 1 (2021): 14-31.

[9] Ghiasi, Emran Khoshrouye, and Reza Saleh. "Analytical and Numerical Solutions to the 2D Sakiadis Flow of Casson Fluid with Cross Diffusion, Inclined Magnetic Force, Viscous Dissipation and Thermal Radiation Based on Buongiorno's Mathematical Model." CFD Letters 11, no. 1 (2019): 40-54.

[10] Sheikh, Nadeem Ahmad, Farhad Ali, Muhammad Saqib, Ilyas Khan, Syed Aftab Alam Jan, Ali Saleh Alshomrani, and Metib Said Alghamdi. "Comparison and Analysis of the Atangana-Baleanu and Caputo-Fabrizio Fractional Derivatives for Generalized Casson Fluid Model with Heat Generation and Chemical Reaction." Results in Physics 7 (2017): 789-800. https://doi.org/10.1016/j.rinp.2017.01.025.

[11] Saqib, Muhammad, Farhad Ali, Ilyas Khan, and Nadeem Ahmad Sheikh. "Heat and mass transfer phenomena in the flow of Casson fluid over an infinite oscillating plate in the presence of first-order chemical reaction and slip effect." Neural Computing and Applications 30, no. 7 (2018): 2159-2172. https://doi.org/10.1007/s00521-0162810-x.

[12] Mahanthesh, B, T Brizlyn, SabirAli Shehzad, and B J Gireesha. "Nonlinear Thermo-Solutal Convective Flow of Casson Fluid over an Oscillating Plate Due to Non-Coaxial Rotation with Quadratic Density Fluctuation Exact Solutions." Multidiscipline Modeling in Materials and Structures 15, no. 4 (2018): 818-842. https://doi.org/10.1108/MMMS06-2018-0124.

[13] Nadeem, S., Rizwan UI Haq, and C. Lee. "MHD Flow of a Casson Fluid over an Exponentially Shrinking Sheet." Scientia Iranica 19, no. 6 (2012): 1550-1553. https://doi.org/10.1016/j.scient.2012.10.021.

[14] Nadeem, S., Rizwan Ul Haq, Noreen Sher Akbar, and Z. H. Khan. “MHD Three-Dimensional Casson Fluid Flow Past a Porous Linearly Stretching Sheet." Alexandria Engineering Journal 52, no. 4 (2013): 577-882. https://doi.org/10.1016/j.aej.2013.08.005.

[15] Khan, Dolat, Arshad Khan, Ilyas Khan, Farhad Ali, Faizan ul Karim, and I. Tlili. "Effects of Relative Magnetic Field, Chemical Reaction, Heat Generation and Newtonian Heating on Convection Flow of Casson Fluid over a Moving Vertical Plate Embedded in a Porous Medium." Scientific Reports 9, no. 1 (2019): 1-18. https://doi.org/10.1038/s41598-018-36243-0.

[16] Mohamad, Ahmad Qushairi, Nurul Aini Jaafar, Sharidan Shafie, Zulkhibri Ismail, and Muhammad Qasim. "Theoretical study on rotating casson fluid in moving channel disk." In Journal of Physics: Conference Series, vol. 1366, no. 1, p. 012039. IOP Publishing, 2019. https://doi.org/10.1088/1742-6596/1366/1/012039.

[17] Alwawi, Firas A., Hamzeh T. Alkasasbeh, A. M. Rashad, and Ruwaidiah Idris. "MHD natural convection of Sodium Alginate Casson nanofluid over a solid sphere." Results in physics 16 (2020): 102818. https://doi.org/10.1016/j.rinp.2019.102818. 
[18] Kumar, K Anantha, V Sugunamma, and N Sandeep. "Effect of Thermal Radiation on MHD Casson Fluid Flow over an Exponentially Stretching Curved Sheet." Journal of Thermal Analysis and Calorimetry 140, no. 5 (2020): $2377-2385$.

[19] Ali, Farhad, Nadeem Ahmad Sheikh, llyas Khan, and Muhammad Saqib. "Solutions with Wright Function for Time Fractional Free Convection Flow of Casson Fluid." Arabian Journal for Science and Engineering 42, no. 6 (2017): 2565-2572. https://doi.org/10.1007/s13369-017-2521-3. 